\title{
Implicit learning of complex structures: Active adaptation and selective processing in acquisition and application
}

\author{
RICHARD L. WRIGHT and BRUCE W. A. WHITTLESEA \\ Simon Fraser University, Burnaby, British Columbia, Canada
}

\begin{abstract}
Subjects exposed to members of a structured domain become sensitive to the general structure of that domain, even when they are unaware that the domain has such structure (e.g., Reber, 1993). Numerous investigators have attempted to characterize this learning as unselective in acquisition and automatic in application. However, we contend that this characterization miscasts the fundamental nature of learning. In a series of experiments, we demonstrate that what subjects learn implicitly about the structure of a domain critically depends on decisions they make about how to organize the structural components. Similarly, the application of knowledge gained implicitly is not stable, but may be selected or even created under the demands of the test task. We conclude that implicit learning, just like explicit learning, proceeds through active organization of the stimulus complex, rather than by passively absorbing any level of structure. We propose a synthesis, in which learning, with and without awareness, is understood through a common set of principles.
\end{abstract}

Many important aspects of the environment, including language, the social matrix in which people live, and the categorical organization of events and objects, consist of complex, abstract rule structures. Within those broad domains, many more specific problem areas, such as the operation of the stock market, the structure of well-formed music or art, or the personality of one's spouse, can also be thought of as complex rule structures. It is therefore of interest to understand how people acquire knowledge about domains defined by complex rules.

Although some of this knowledge may be acquired through formal communication from mentors or through explicit, deliberate generation and testing of hypotheses about the world, much of it appears to be acquired without people being aware that there is a structure to be learned, or even that they are learning. For example, children learn to produce comprehensible utterances, fluently and competently. In effect, they learn to use the complex structure of English syntax to achieve social goals. This learning occurs with much practice and with much informal feedback (the effect that their utterances have on others), but it is performed incidentally to the satisfaction of other objectives and without the explicit intention to learn the rules of language. Moreover, although this learning enables children to use language, it is not accompanied by conscious aware-

This research was supported by a grant to B.W.A.W. from the Natural Sciences and Engineering Research Council of Canada. We gratefully acknowledge the assistance of Susan Cook. Correspondence should be addressed to B. W. A. Whittlesea, Department of Psychology, Simon Fraser University, Burnaby, BC, Canada V5A IS6 (e-mail: bruce_ whittlesea@sfu.ca).

-Accepted by previous editor, Geoffrey R. Loftus ness either of what the rules of language are or even that language is a rule-governed activity: Children still have to be taught the rules of syntax formally in school. However, this explicit instruction is not what is responsible for their ability to use language. In fact, children cannot be taught the rules explicitly until they already know language well enough to understand the spoken explanation.

This phenomenon of performance that is sensitive to underlying rules, without conscious knowledge of those rules, is typical of people's interactions with complex domains. People are often much more able to interact with their environment than they are able to explain. Explicit knowledge of the deep structure of a rule-governed domain is unnecessary for efficient performance, and much of what people know about such domains is learned implicitly, without becoming aware of the underlying structure.

Such implicit learning has been demonstrated in a wide range of situations, including learning artificial grammars (e.g., Reber, 1969, 1976), controlling dynamic systems (e.g., Berry \& Broadbent, 1988), detecting static invariances (e.g., Lewicki, 1986; McGeorge \& Burton, 1990), and becoming sensitive to the probability of events in structured sequences (e.g., Nissen \& Bullemer, 1987). For instance, in the artificial grammar task, letter strings (e.g., MXRVXT and VXVTRRX) are generated by a complex set of rules. Subjects are exposed to these strings without being informed of the method of generation, and then, in a surprise test, they are asked to categorize novel strings as either rule following or rule violating. Reber (1976; Reber \& Allen, 1978) observed that subjects' ability to discriminate between legal and illegal test strings was above chance, but that they were generally unable to explain the basis for their success. Subjects had apparently become sensitive to the structure of the set, even though 
they were unaware that the domain possessed a deep structure.

The knowledge acquired when people learn implicitly appears to be quite different from that which they acquire when they attempt to explicitly test hypotheses about the deep structure of a domain. Ordinarily, when encountering a novel domain that they know to be rule governed, people attempt to deduce the rules through explicit generation and testing of hypotheses about the underlying rule structure. Successful discovery of the true underlying rules grants the person complete control of the domain, but failure to discover the rules leaves him or her with no useful knowledge (Berry \& Dienes, 1993). This explicit learning is deliberate and effortful, governed by the general intention to learn about the deep structure, and by momentary, specific intentions to test particular aspects of that structure. Because the act of learning is under the conscious control of the learners, the resulting knowledge will depend on the processes they decide to engage during encoding. The essential characteristic of explicit learning is that it is flexible and variable: At will, the learner can selectively process a stimulus in this way or that, focusing on this or that attribute, and compare, contrast, and compute abstract information about similarities or differences among stimuli. The structure of the stimuli constrains these activities, but the person has the freedom to process that structure in a wide variety of ways. Such learning can thus be expected to be highly variable, and will be unpredictable by an observer, even when the domain is held constant.

In contrast, implicit learning seems to be an effortless and automatic process. It is thought to occur when the learner is unaware that the domain is rule governed and therefore does not attempt to generate hypotheses. Nonetheless, people do acquire some knowledge about the domain that enables them to be robustly but imperfectly sensitive to the underlying rules of the domain. However, because the subjects are unaware of the existence of deep structure, their learning about that structure is not modulated by consciousness. Instead, it is driven primarily, or exclusively, by the structure of the incoming stimuli. The learner is in essence a passive recipient of knowledge (Berry \& Dienes, 1993; Lewicki, 1986). Thus, for example, Hayes and Broadbent (1988) characterized implicit learning as an "unselective and passive aggregation of information about the co-occurrence of environmental events and features" (p. 251): Cleeremans (1993) noted that "a hallmark of implicit learning processes ... is that they proceed in an unintentional way ... any structure that emerges as the result of [implicit] processing must be almost entirely stimulus-driven" (p. 19). Whatever the mechanism by which learning proceeds, it must consist of automatic memory processes that can proceed without modulation by consciousness or intention. In consequence, such learning must be unselective, passive, stable, and inflexible: Variations in learning will result only from variations in the structure of successive stimuli.
Learning in this way reveals a natural and automatic tendency of the brain to absorb the structure of the world (Lewicki \& Hill, 1989) and to adapt passively to that structure (Reber, 1993): It is at once more powerful but less flexible than explicit learning as a means of becoming sensitive to the deep structure of complex domains.

This characterization of implicit learning caused many investigators to assume that it represents a different mode of learning than explicit learning and to search for a mechanism whereby memory could absorb the structure of a domain without the person being aware that it exists. The dominant account has been that implicit learning consists of acquisition of direct knowledge about the deep, general structure of the domain. In this account, memory abstracts information across successive stimuli and thus acquires knowledge about the co-occurrence of features across stimuli (e.g., Cleeremans, 1993; Knowlton \& Squire, 1994; Mathews, Buss et al., 1989; Mathews, Druhan, \& Roussel, 1989; Reber, 1969, 1989, 1993). Numerous mechanisms have been proposed that would enable memory to compute some knowledge about abstract structure without supervision, including competitive chunking (ServanSchreiber \& Anderson, 1990), classifier systems (Mathews, Druhan, \& Roussel, 1989), and, more recently, connectionist architectures (Cleeremans, 1993; Dienes, 1992). These mechanisms differ in the level of abstractness at which implicit learning is thought to operate, but all assume that it is a passive, stimulus-driven activity, occurring through the operation of automatic memory processes. Because learners are not aware of the existence of the structural regularities to which they develop sensitivity, it is assumed that this learning is not dependent on or influenced by conscious thought or intention. Thus the task that the learner performs on the stimuli is irrelevant as long as it permits the subject to experience the stimuli fully. Moreover, because it is driven only by the structure of the stimuli, this learning and its products will be unselective, representative of the objective distribution of occurrence and co-occurrence of features in the domain.

The idea of an autonomous learning mechanism that automatically computes the abstract structure of a domain offers a direct solution to the puzzle of implicit learning. However, we believe that this approach oversimplifies the fundamental nature of both explicit and implicit learning. It rests on three important assumptions, all of which we believe to be misguided.

Assumption 1: In the absence of explicit hypothesis testing, acquisition of knowledge is directly determined by the structure of the domain. That is, when exposed to a domain containing hidden deep structure, people may become actively involved in trying to manipulate or learn the stimuli, but this involvement is not what is responsible for the form or content of the effective knowledge base they will acquire about the domain. Instead, that learning is dictated by the proclivity of the mind to process abstract properties of structure in the world. In consequence, the extensiveness of people's implicit sen- 
sitivity to abstract structure is determined primarily by the variability of the structure that they encounter.

In contrast to that assumption, we believe that the absence of explicit hypothesis testing and the lack of awareness of deep structure to search for do not make the learner a passive or unselective recipient of structure. The subject is still actively adapting to the circumstances, exercising learning strategies and making processing decisions based on a host of situational and historical factors (see Whittlesea \& Wright, 1997). These decisions will critically determine the perceptual and cognitive operations subjects will impose on the stimuli, and in consequence will determine what they learn about the stimuli and what aspects of structure they will be sensitive to in a subsequent test. The learning that occurs without awareness of deep structure is as open to variation and selectivity as if subjects were explicitly testing hypotheses. We argue that such variability is not usually observed in implicit learning studies simply because the investigators usually do not vary the induction task. Obviously, if that task is held constant, its influence cannot be measured-but that does not mean that what the subjects learn is not controlled by the specific demands of that task. When subjects are given different induction tasks, the extent of sensitivity to implicit stimulus properties varies tremendously (see Vokey \& Brooks, 1992; Whittlesea \& Dorken, 1993; Whittlesea \& Wright, 1997).

Assumption 2: Knowledge acquired implicitly provides a stable knowledge base that is automatically and consistently applied at test. According to the first assumption, memory absorbs some particular unit(s) of structure, independent of conscious intention. It follows that if a subject is exposed to further stimuli bearing the same structural organization, memory will respond automatically to those same structural units. In consequence, providing a set of test stimuli differing on some structural dimension can be used as a neutral index to determine whether the subject had previously acquired knowledge of that aspect of structure.

In our view, memory does not simply acquire knowledge about some salient structural units and automatically apply that knowledge whenever those units reappear in a subsequent task. Instead, the original acquisition, and equally the application of knowledge, is controlled by subjects' decisions about how to interact with current circumstances. Each test task presents some demand on the subjects: Subjects will respond to that demand by processing the stimuli in some particular way, different from the way in which they would have performed under some other test. The structure of test stimuli constrains the variability of performance but does not unilaterally determine what knowledge the subjects will apply. Rather than simply revealing what a subject learned previously, the test task can cause subjects to reorganize their previous knowledge and compute additional information out of the knowledge they already have.

Assumption 3: Sensitivity to an abstract structural property indicates that people have computed abstract knowledge about the domain. The standard implicit learning study exposes subjects to a list of items possessing some general, abstract property and then presents test items, half of which preserve that property and half of which violate it. The observation that subjects can discriminate between the sets is often taken as presumptive evidence that they have acquired knowledge about that property; when subjects are not able to report knowledge of that property, they are presumed to have learned it implicitly. Further, because the property is abstract, consisting of a relationship between training stimuli, the knowledge is presumed to be acquired through a process of abstraction, automatically accumulating information about the covariation of features across the set.

This assumption presents a false dichotomy-that if sensitivity to an abstract property is not achieved explicitly, through deliberate hypothesis testing, then it must be achieved unconsciously, through automatic abstraction of regularities across stimuli. The fallacy of this reasoning has been demonstrated repeatedly (e.g., Brooks, 1987; Cock, Berry, \& Gaffan, 1994; Vokey \& Brooks, 1992; Whittlesea \& Dorken, 1993; Wright \& Burton, 1995). People need not have direct knowledge of a property to respond discriminatively in its presence and absence. General properties of the set are not independent of the properties of individual instances of the set: Any test item that violates a property that is true of the set as a whole will also present a combination of features different from those present in individual training items. Numerous authors have demonstrated that subjects can be sensitive to deep, abstract properties of the domain if they have only encoded some aspects of the surface structure, such as the identities of the presented instances (Brooks, 1978), fragments of the instances (Dulany, Carlson, \& Dewey, 1984), or merely bigrams from the instances (Perruchet \& Pacteau, 1990, 1991). Thus the knowledge that makes the subject able to discriminate legal from illegal test stimuli may not be direct knowledge about the abstract property, but instead some knowledge about specific training instances that is correlated with that abstract property.

If the basis of implicit learning is simply acquiring knowledge about individual training stimuli, then the acquisition of that knowledge does not require any unconscious abstraction mechanism. Moreover, the act of learning itself is not implicit: The subject learns about individual stimuli with full awareness. The application of that knowledge in the test may be equally explicit: Subjects judge stimuli on the basis of item information of which they are aware. What is implicit, what subjects are not aware of, is the correlation of their knowledge with abstract properties of the domain. That is, subjects implicitly acquire the potential to respond discriminatively on dimensions of which they are unaware at the time of learning and of which they may continue to be unaware at the time they perform the discrimination by explicitly learning correlated knowledge about individual instances. This learning has the effect of enabling subjects to be sensitive to the presence or absence of the abstract property without ever learning directly about it or knowing that it exists. 
Despite the abundance of demonstrations of this point, the idea that sensitivity to an abstract property reflects the possession of direct knowledge about that property still retains the status of the null hypothesis, to be freshly disproven each time implicit sensitivity to deep structure is observed (see, e.g., Knowlton \& Squire, 1994; Reber, 1993). One of the purposes of the present experiments is to reverse that onus by demonstrating that subjects always learn about the training stimuli in a manner dictated by the purpose of the induction task and that sensitivity to an abstract property emerges only when that task causes subjects to compute information that is correlated with the abstract property. That is, we will attempt to demonstrate that to produce implicit sensitivity to an abstract property of the training stimuli, it is necessary and sufficient that the training task require subjects to compute information that is correlated with that property. If we succeed in that objective, it is then up to others to demonstrate that in addition to that knowledge, subjects also acquire some direct, unconscious knowledge about that property, independent of the demands of the induction task.

Fundamentally, we argue that there is only one kind of learning. People respond adaptively to the demands of tasks and the affordances of stimuli by processing stimuli in whatever way is functional to satisfy current objectives; memory preserves whatever the person experiences in the course of satisfying current purposes. This is true regardless of whether the task requires memorization of stimuli, hypothesis testing, or backward reading, whether the domain does or does not possess deep structure, and whether people explicitly think of their current interaction with stimuli as an occasion of learning or simply the execution of a demand. Depending on the task, the learning may be more or less focused and extensive, and the person may be more or less concerned with performing an action to prepare for the future or to complete a present demand; different purposes cause the person to process different stimulus aspects, in different relationships. However, the consequence of interacting with stimuli is always the same: people acquire a representation of whatever they experienced in processing a stimulus for the current purpose.

These encoded processing experiences serve as resources for processing further stimuli, on other occasions and in other tasks. Performance in those later tasks succeeds to the extent that earlier experiences have provided the specific resources necessary to accomplish those tasks. In cases that are called "explicit learning," the earlier experiences may be directly related to the subsequent demand, in which case people may recognize the source of their ability to perform the later task (even if they did not anticipate that task when performing the original learning). Alternatively, those experiences may be only indirectly and even accidentally related to the later task, and the person may not realize the source of their ability; those cases are called "implicit learning." Regardless of people's awareness of the source of their ability, and even regardless of their awareness of their success in performing a task, the principle controlling later performance is always the same: Performance succeeds to the extent that resources exist to perform the activity and are cued by the demands of the task. We therefore argue that there are not two forms of learning - "explicit" and "implicit"-but instead that there is only learning, and that people may be aware or unaware of the implications of that learning for the future and aware or unaware on a later occasion of the source of their ability to perform.

The experiments reported here were designed to demonstrate this view of learning. Experiment 1 demonstrates that the orientation task of an implicit learning experiment does not serve merely as an opportunity to experience the structure of the domain, but instead has a major role in determining what aspects of structure are processed. Experiments $2-4$ show that implicit sensitivity to a structural invariant occurs only when the induction task causes subjects to compute information correlated with the invariant. Experiment 5 demonstrates that the effective knowledge used to discriminate among test items need not have been actually computed prior to the test; instead, subjects may acquire some knowledge about training items and compute additional information from that knowledge in response to the particular demands of the test. Together, the experiments show that "implicit learning" is not the automatic acquisition of knowledge about abstract properties of experience. Instead, it is the acquisition of the potential to respond to an unanticipated demand, using knowledge that is only indirectly related to that demand, that was computed directly to satisfy the demands of the induction task.

\section{PART I \\ The Nonneutrality of Training Tasks}

The usual training procedure of an implicit learning experiment is to expose subjects to members of the stimulus set without making them aware of the structured nature of the domain. To expose the training stimuli without inviting hypothesis testing, stimuli are presented to the subject in an induction task (sometimes referred to as a distractor or orientation task). For example, in artificial grammar learning experiments, subjects are usually asked to memorize letter strings for a later memory test. Although the subjects obviously learn something specific about individual stimuli in performing this task, the "automatic abstraction hypothesis" assumes that this explicitly acquired knowledge is not the basis of their later sensitivity to general properties of the domain. Instead, the induction task is important only as an opportunity for unconscious abstraction processes to operate, producing additional, implicit knowledge about the general structure of the domain. Under this assumption, the induction task is neutral with respect to the development of implicit sensitivity to general structure.

However, that assumption of neutrality is valid only if implicit learning actually does consist of the abstraction of general structure across stimuli, independent of what 
the subjects compute about the structure of individual stimuli. In contrast, we contend that implicit learning consists exactly of acquiring knowledge about particular stimuli under the control of the induction task, without any additional implicit abstraction; the sensitivity to global properties of the domain observed in a later test results from the correlation of those properties with the information that subjects acquired explicitly. We demonstrate this contention in Experiments 2-5. Under that understanding of implicit learning, the demands of the induction task are certainly not neutral because they determine what people will learn about particular stimuli and hence determine their later sensitivity to abstract properties of the domain.

\section{Experiment 1 \\ The Instability of Structural Organization}

Before examining the development of sensitivity to the general structure of a domain, we need to clear up one point: There is no such thing as an induction task that is neutral with respect to encoding the structure of individual stimuli. People inevitably impose organization on the structure of the stimuli they attend. That organization varies with a number of factors, including the familiarity of the stimuli and their components, the instructional demands of the task, the perceptual manifestation of the stimuli, the operations necessary to satisfy the current purpose of the encounter performed on the stimuli, and the relative ease of encoding alternative organizations. People react to these factors by isolating or grouping stimulus features, weighting them equally or differentially, and processing them in selective sequences. They are never in the position of simply absorbing the objective structure of stimuli.

To illustrate that idea, we asked subjects which of two four-digit numbers (e.g., 6491 and 1537) was more similar to a third (e.g., 6537). This comparison can be made at either of two levels of abstraction: 6491 and 6537 are more similar if the stimuli are treated as whole numbers, whereas 1537 and 6537 are more similar if stimuli are treated as lists of separate digits. We did not instruct subjects how to think about the stimuli or perform the comparison, or ask them to do anything else with the numbers. Our only manipulation was to present the numbers side by side for some subjects, and one on top of another for the remaining subjects (see Figure 1).

The groups received identical triplets of items. The internal structure of the stimuli and the formal structural relationships between targets and comparators were thus also identical for both groups. The instructions to the groups were identical. The only difference between conditions was the spatial arrangement of stimuli, and that factor is irrelevant to their individual or set-wise structure. If people are ever passively and unselectively sensitive to formal properties of structure, then they should be so under these circumstances. In that case, the subjects' judgments of similarity should be controlled by the true, objective similarity of the stimulus structures, and there should be no reliable difference between groups.

However, the spatial arrangement affects the relative ease of treating the stimuli as consisting of a single unit or four separate units. Given side-by-side presentation, it is easier to compare the outside items to the middle item if one encodes each as a four-digit number. In contrast, given vertical presentation, it is easier to compare the outside items to the middle item one digit at a time. If that processing actually causes subjects to organize the structures of stimuli at different levels of abstraction, then we could expect a difference in judged similarity between the groups.

\begin{abstract}
Method
Subjects. Eighteen Simon Fraser University students participated in this study for course credit.

Materials. Ten sets of four-digit numbers were generated, each set containing three numbers, a target and two comparators. For example, one set contained the target 9541 and the comparators 9631 and 2541 . The sets were contrived so that the first comparator was more similar to the target if it was interpreted as a single four-digit number, "nine thousand five hundred and forty-one." The second comparator was more similar to the target if it was treated as made up single digits-9,5,4, and 1 . The second comparator and the target always had three out of four digits in common while the first comparator had only a one- or two-digit overlap with the target.

Procedure. The same stimulus triplets were presented in two conditions. Half of the subjects participated in one condition, half in the other. In both conditions, the target item was presented in the center of the screen. In one condition, the comparators were presented one on each side of the target (separated from the target by a gap of $20 \mathrm{~mm}$ ); in the other, one was presented $4 \mathrm{~mm}$ above and one $4 \mathrm{~mm}$ below the target. In both conditions, subjects were told
\end{abstract}


that they would see three four-digit numbers on each trial. They were asked to indicate which of the outside numbers (depending on condition, this would be top or bottom, or left or right) was more similar to the number in the middle. They were informed that we were not interested in how fast they could do the judgment, but simply wanted to know which was more similar.

\section{Results and Discussion}

In principle, subjects could have based their similarity judgments either on the numerical similarity of the items or on the match of features. When the items were presented vertically, subjects usually chose the feature-match stimulus ( $p=.81 ; S D=.21)$; when the items were presented horizontally, however, they rarely chose the featurematch item $(p=.30 ; S D=.40)$, instead choosing the whole-number-match item $[t(16)=3.42, p<.01]$. We concluded that vertical presentation made the subjects more likely to encode the target as four separate digits, whereas the side-by-side presentation promoted processing it as a single, whole number. The mode of spatial presentation thus induced a change in the level of abstraction at which subject processed the items, independent of their formal structure. We concluded that subjects in each group had imposed an organization on the structures of the stimuli as a functional adaptation to the specific presentation conditions. We take this as an illustration of a general principle - that regardless of the overt task or purpose for which stimuli are encountered, people always impose some organization on their structure.

This experiment helps to clear up a concept that has complicated the study of implicit learning. As discussed earlier, numerous authors have described such learning as "stimulus driven." This description suggests that stimuli possess some raw, objective structure and that there is a mode of learning in which the subject's processing is directly controlled by that structure, independent of the subject's task or intentions. The present data demonstrate how that mistaken interpretation can come about. Consider only the first group of subjects. Those subjects consistently responded to the similarity of stimuli taken as separate digits. Because the subjects all had the same task, intention, instructions, and so on, those factors could not be responsible for the difference in how they treated the two kinds of comparator, favoring the digit-match item over the whole-number-match item. Instead, that difference must be due to the difference in structural similarity between the target and the two comparators. The subjects' processing was thus clearly stimulus driven.

However, the other group, also considered in isolation, leads to the same conclusion. For them, as well, task, intention, and presentation were held constant, and only structural differences between the target and comparators could account for their consistent preference for the wholenumber-match item. Both groups, considered alone, demonstrate stimulus-driven processing, and yet their performance on the same stimuli was qualitatively different. This illustrates the inadequacy of the concept of stimulusdriven processing. The concept really only means that if people's active organization of stimuli is held constant at some level by holding the task constant, then the residual variance in performance can be accounted for only through differences in the stimulus structure. It does not mean that in any given task, the subjects' intentions and the demands of the task are not critical in determining their processing, or what they learn about the stimuli. When a factor other than the structures of the stimuli (the perceptual array) was also varied, it became obvious that subjects in both groups had imposed a particular organization on the stimuli, and that organization dictated the level at which the structure of the stimuli would be processed. Fundamentally, we argue that the structure of stimuli never has unilateral or even dominant influence. Performance always results from an interaction between the structure of the stimuli and the organization that people impose on that structure in the current circumstances.

The concept of stimulus-driven processing has been a major obstacle to a clear understanding of implicit learning. Most experiments used to support the "automatic abstraction hypothesis" have used a single induction task. Under those conditions, differences in how subjects process the structures of stimuli could not manifest themselves, and the vital role of the subject adopting a taskappropriate way of processing the stimuli was invisible. We will return to this issue in Experiment 3.

\section{PART II Processing-Appropriate Transfer}

McGeorge and Burton (1990) showed subjects fourdigit numbers, each of which contained a 3 and three other digits. Subjects were not informed about the existence of the invariant 3 , but were asked to perform a simple arithmetical operation on each of the training items. This task involved a comparison between the sum of the two digits on the left (first and second digits) with the two on the right (third and fourth digits). In a subsequent recognition test, subjects were given pairs of four-digit numbers and asked to identify one member of each pair as "old" (i.e., presented earlier in the training phase). ${ }^{1}$ In fact, unknown to the subject, all stimuli at test were novel; however, one item contained a 3 and the other did not. McGeorge and Burton observed that subjects identified more items containing a 3 as familiar, even though none could report the "invariant 3 " rule. These results demonstrate sensitivity to the underlying structure of the training set, in the absence of verbalizable knowledge of that structure.

McGeorge and Burton's (1990) induction task required the subjects to compute aspects of the stimuli that were not directly related to the abstract invariant. ${ }^{2}$ They therefore assumed that their induction task simply offered an opportunity to observe the structure of the training stimuli and was not itself responsible for the subjects' sensitivity to the invariant 3 in test. However, like many other investigators, they did not test that assumption by varying the induction task.

In the experiments below, we replicated the phenomenon of implicit learning. That is, we observed that follow- 
ing experience of a set of stimuli, subjects became sensitive to an abstract property of the set without becoming aware of the existence of that property. However, we also observed that such implicit sensitivity is not an automatic consequence of exposure to the set (Experiment 2) and that it does not occur through direct computation of the abstract property (Experiment 3 ). Instead, we observed that such implicit sensitivity occurs only when the induction task leads subjects to compute information that is correlated with the abstract property (Experiments 3 and 4).

\section{Experiment 2}

\section{The Bounds of Implicit Learning}

Before investigating the mechanism of implicit learning per se, we first examined the boundary conditions of the phenomenon. In Experiment 2A, we showed subjects training stimuli containing two abstract invariants: (1) The sum of the first two digits (the "left" digits) always equaled the sum of the third and fourth digits (the "right" digits), and (2) the sum of the second and third digits (the "inside" digits) was always less than the sum of the first and fourth digits (the "outside" digits). Examples of such items include 7328 and 8154.

We also used two kinds of induction tasks in the training phase. Half the subjects compared the sum of the first two digits with the sum of the last two, and the other half compared the sum of the inside digits with the sum of the outside digits. All subjects were then given a surprise test in which they were asked to identify the "old" fourdigit number in each of 10 pairs (in line with previous studies, no test items were actually repeated from training). One item in each pair preserved the first invariant and violated the second; the other item preserved the second invariant and violated the first.

\section{Experiment 2A}

\section{Method}

Subjects. Twelve undergraduate students attending Simon Fraser University participated in this experiment for course credit.

Materials. Thirty training items were generated according to the constraints that the sum of the left and right pairs of numbers were equal and that the sum of the inside pair of numbers was less than the sum of the outside pair. None of these items contained repeated digits. These invariant forms of information were abstract in the sense that they were true of every stimulus, although the actual sums varied across stimuli.

Each training item was used to generate two test items. One was created by interchanging the inside digits, and the other by interchanging the larger of the outside digits with the digit adjacent to it (e.g., the training item 8246 produced the test items 8426 and 2846). The first type of item only preserves the relationship that the sum of the inside numbers is less than the sum of the outside digits; the other only preserves the equality of the sums of the left and right pairs. At test each subject saw 10 pairs of four-digit numbers. A test pair consisted of one item preserving each of the invariants, although test items generated from a single training item were not shown together.

Procedure. Subjects were randomly assigned to one of two training conditions. All subjects were told that they would see fourdigit numbers. Half the subjects were asked to mark these with a " + " when the sum of the first two digits was greater than the sum of the second two, a "- "when the sum of the first two was less than the sum of the second two, and to leave the item blank if the sums were equal. The other half of the subjects were given similar instructions, but were asked to compare the sum of the inside digits (second and third) with the sum of the outside digits (first and fourth).

At test, subjects were shown pairs of four-digit numbers and (falsely) told that one number in each pair had been shown in the previous phase of the experiment. They were asked to indicate which one this was, guessing if they were not sure.

\section{Results and Discussion}

The test offered subjects a choice between two stimuli, each of which preserved one of the abstract invariants that had been present in the training stimuli. On $98.3 \%$ of trials, the subjects selected whichever item preserved the invariant they had been led to process in training, reliably greater than chance $[t(11)=26.50, p<.001]$. We concluded that the subjects had become selectively sensitive to the invariant related to their task, but had done so explicitly: In performing repeated calculations under the demands of the task, they had become aware of the abstract relationship between the parts of the stimuli they were required to compare. We concluded that we had exceeded one boundary of implicit learning. When the information computed under the induction task is directly related to an abstract property of the domain, people will become explicitly aware of that property.

In Experiment 2B, we asked subjects to perform the same two addition tasks as in Experiment 2A. We embedded a single abstract invariant in the training set: The last two digits always differed by one, the last always being smaller than the second last (e.g., 65, 54). (Actually, there was another invariant relationship that subjects could learn about: Because of the different-by-one rule, the last two digits always differed in terms of being even or odd.) In this case, unlike Experiment $2 \mathrm{~A}$, the abstract invariant was not directly related to the computation that subjects performed on the stimuli. However, this relationship is much simpler than the ones used in Experiment 2A: Unlike those invariants, which cannot be discovered without extensive computation, the smaller-by-one invariant can be detected by inspection. The question was whether the subjects would become sensitive to an invariant that was not related to the information generated in performing the induction task, and if so, whether processing the two last digits together or in separate operations would influence the degree of later sensitivity to their relationship.

\section{Experiment 2B}

\section{Method}

Subjects. Twenty undergraduate subjects attending Simon Fraser University participated in Experiment 2B, for course credit.

Materials. Twelve training items were generated, subject to the constraint that the last two digits differed by one and that the last was smaller than the second last. The digit pairs $76,65,54$, and 43 were each used in the terminal locations of three items; the digits occurring in the first two positions were selected at random, subject to the constraint that no item contained repeated digits (e.g., 8165).

Eight pairs of novel items were used in test. One member of each pair preserved the descent-by-one pattern in its third and fourth lo- 
cations: The digit pairs $76,65,54$, and 43 each occurred in two items. The other two digits were selected so that the combination had not been shown in training, no item contained repeated digits, and their sum was the same as the sum of digits in those locations in at least one training item (e.g., 7265). The other member of each pair violated the descent-by-one pattern; otherwise, its digits were selected subject to the same requirements as the first set.

Procedure. Half of the subjects were asked to add the first pair of digits in each training stimulus, as well as the last pair; the other half added the inside pair of digits, and also the outside pair. At test, all subjects were (falsely) told that one member of each test pair had been seen in training and were asked to identify the old member of each pair.

\section{Results and Discussion}

Overall, subjects claimed to recognize target items (containing the invariant descending-by-one pattern) on $49 \%$ of trials, against the chance rate of $50 \%[t(19)<1]$. The specific induction task made little difference. Subjects required to add the last two digits together selected target items on only $45 \%$ of trials, and subjects who had processed those digits in separate addition operations selected target items on $53 \%$ of trials; neither was reliably different from chance $[t(9)<1$ in both cases], nor were they reliably different from each other $[t(18)=1.2]$. We concluded that there was no evidence that subjects had acquired any ability, explicitly or implicitly, to discriminate items preserving the invariant pattern from those violating it.

This failure demonstrates that sensitivity to an abstract invariant is not an automatic consequence of processing stimuli extensively. Instead, that sensitivity appears to depend on the relationship between the invariant and the knowledge the subject computes under the control of the induction task. In Experiment 2A, the information that subjects were required to compute (the sums of digit pairs) was directly related to the abstract invariant (the equality of sums of pairs); although not asked to compare the sums of the digit pairs, those subjects became explicitly aware of the invariant relationship. In Experiment 2B, the information that subjects were required to compute (again the sums of digit pairs) was unrelated to the invariant (the constant difference of the terminal digits, or their even-odd relationship); those subjects did not become sensitive to the invariant. We suspect that these are examples of a general principle - that people only learn about those properties of their experience that they are led to process to satisfy the task or that are systematically related to their task, and do not automatically and unconsciously compute abstract properties of the domain that are irrelevant to their task. ${ }^{3}$

How, then, do people become sensitive to abstract stimulus properties without becoming aware that they exist? We suggest that people do not directly learn about those properties. Instead, in the course of satisfying the induction demand, they learn some information that is correlated with the implicit property. In that case, the subjects could discriminate test items preserving an implicit in- variant from those violating the invariant, without ever realizing that the invariant exists, but they would do so using a different form of information. That is, the subjects become accidentally sensitive to the invariant, through its correlation with properties that they have directly processed under the control of the induction task. We illustrated this principle in the next experiment, showing that subjects can become sensitive to an abstract invariant without awareness, but only if they are directly required to process some information that is indirectly related to that invariant.

\section{Experiment 3 \\ Implicit Sensitivity as an Incidental By-Product of Explicit Learning}

Experiment 3 was designed to demonstrate a case of truly implicit learning in which subjects actually become sensitive to an abstract invariant without becoming aware of its existence. However, the experiment also shows that this sensitivity does not occur through automatic abstraction of the invariant, occurring independently of the demands of the induction task. Instead, that sensitivity is a by-product of computing information to satisfy those demands. Specifically, this sensitivity demonstrates that (1) different induction tasks cause subjects to compute different types of information about the stimuli; (2) subjects only compute information of direct relevance to their task, and do not unconsciously compute information about structural invariants that are not relevant; but (3) the information that subjects compute directly, in response to the demands of the task, can make them indirectly sensitive to such invariants, producing the phenomenon of "implicit learning."

To illustrate these points, we created a set of 16 fourdigit numbers, each of which followed the pattern oddeven-odd-even (e.g., 1834). This invariant odd-even pattern was the implicit rule of the set. Subjects were never told about the existence of this invariant, either in training or in test, and the induction tasks did not require subjects to notice or compute any information about evenness or oddness. The question was whether subjects exposed to these stimuli would become sensitive to that invariant without becoming aware of it, and if so, whether that sensitivity occurred through an autonomous, unconscious abstraction process or, alternatively, because the induction task led the subjects to compute information that was indirectly related to the invariant.

To discover whether the subjects' sensitivity to an invariant depends on the specific nature of the induction task, we varied that task between groups. One group was asked to read each number aloud, pronouncing each as a pair of two-digit numbers (e.g., to read 1258 as "twelve fifty-eight"); the other was to read each number as four separate digits (e.g., "one-two-five-eight"). Both of these tasks expose the subject to the entire structure of each stimulus; thus, if implicit learning consists of automatic 
abstraction of general properties of the domain, we would expect subjects to become as sensitive to the odd-even rule in one condition as in the other. However, if the knowledge that supports sensitivity to implicit properties of the domain actually consists of information directly and explicitly computed to satisfy the demands of the induction task, then we would expect differential sensitivity to the implicit invariant after the two types of training.

The latter prediction is predicated on the idea that the two induction tasks actually caused subjects to encode the stimuli in different ways. We checked that in Experiment $3 \mathrm{~A}$. We then tested the major hypothesis of the study, that differences in the induction task would alter the subjects' sensitivity to the implicit invariant, in Experiment 3B, presented later.

\section{Experiment 3A}

\section{Method}

Subjects. Twenty undergraduate students attending Simon Fraser University participated in Experiment 3A, for course credit.

Materials. To construct stimuli for the induction phase, we created a pool of bigrams, each consisting of an odd digit followed by an even digit, using only the digits from 1 to 8 . The set of bigrams used consisted of $\{12,16,34,38,52,58,74,76\}$. Each bigram was combined with four others to create four-digit stimuli. Each bigram occurred twice in the first half of a stimulus (e.g., 1258 and 1234) and twice in the second half (e.g., 3812 and 7412). No digit was allowed to occur twice in any stimulus. This process generated 16 stimuli. Across these stimuli, each single digit occurred at two stimulus locations (depending on whether it was even or odd), and with the same frequency as all other digits.

Two types of stimuli were generated for test. The first type was created by reversing the bigrams of half the training items; for example, 1258 produced 5812 , and 7412 produced 1274 . Each bigram from the training was used twice, once in the first half and once in the second half of a test item. This produced eight stimuli, consisting of the same bigrams used to create training stimuli, but presenting those bigrams in a novel combination.

The second set of stimuli was created using a set of bigrams that had not been used earlier, namely $\{14,18,32,36,54,56,72,78\}$. Each was used twice, once in the first half and once in the second half of a test item, again producing eight stimuli. Although this set consisted of bigrams that were novel at test, they consisted of the same set of single digits (from 1 to 8), presented at the same locations and with the same frequency, as in the previous set. The only difference between stimulus types was the familiarity of their component bigrams from the training phase.

Procedure. In a training phase, subjects were shown the 16 training stimuli, one at a time, in random sequence, and asked to memorize each for a later test. One group was instructed to perform the memorization by saying each number aloud, reading it as two twodigit numbers (e.g., 1258 read as "twelve, fifty-eight"). The other group was instructed to read each number aloud as a string of separate digits. In addition, the other group was asked to judge each number as low or high, depending on whether it was 5 or larger (e.g., 1258 read as "1-low-2-low-5-high-8-high"). Both groups were allowed $5 \mathrm{sec}$ to rehearse each item and were given two passes through the stimuli.

At test, subjects were told that they would see some new stimuli and some stimuli repeated from the training and were asked to indicate which they had seen before. The 16 (actually all novel) test items were presented, one at a time, in randomized sequence.

\section{Results and Discussion}

The two induction tasks were intended to cause subjects to encode the numbers differently, as digit pairs and as lists of individual digits, respectively. This experiment served as a manipulation check, permitting us to evaluate whether the two types of induction task really had caused subjects to encode the stimuli differently. Subjects who had only encoded information about individual digits (including the location in which those digits occurred) would be unable to discriminate between the two types of test items because the two types presented the same digits in the same locations with equal frequency. In contrast, subjects who had encoded specific pairs of digits presented by training items would be able to discriminate between the two types of test items because one presented those same pairs (recombined to make new items) and the other presented novel pairs. In effect, these subjects could claim to recognize test items by recognizing their parts. Knowledge about the odd-even rule, whether acquired directly or indirectly, was not an issue in this test because all test stimuli followed that rule.

We observed that subjects asked to read training items as single digits could discriminate the two types of test item to some degree. They claimed to recognize $56 \%$ of items consisting of old digit pairs and rejected $59 \%$ of items containing new pairs. Treating the former as hits and the latter as correct rejections, they achieved an overall $57.5 \%$ ability to discriminate the two types of item, reliably above chance $[t(9)=2.88, p<.018]$. We concluded that this group had acquired some knowledge about bigrams in spite of our attempt to make them process training items as separate digits. ${ }^{4}$

However, we also observed that the other group of subjects, who read training items as two pairs of digits, were better able to discriminate the two types of test items. They accepted $64 \%$ of items consisting of old pairs and rejected $78 \%$ of items containing new pairs. They thus achieved an overall $71 \%$ ability to discriminate the two types of items, reliably greater than the first group $[t(18)=2.36, p<.030]$. We concluded that this group had learned more extensively about the bigrams of training items than had the first group. We also noted that both groups achieved discrimination between the types more by rejecting items containing new bigrams than by accepting items containing familiar bigrams.

Now that we knew that the induction tasks caused subjects to process and encode different information about the training stimuli, the question was whether this difference would have any effect on their sensitivity to the odd-even rule. According to the "automatic abstraction hypothesis," the different induction tasks should have no effect on discrimination of legal from illegal items: Both tasks should merely serve as opportunities to observe and abstract the invariant properties of the domain. In contrast, if subjects discriminate legal from illegal items by accepting familiar bigrams and rejecting unfamiliar ones, 
then the more extensive bigram encoding of the bigramreading group should enable them to discriminate legal from illegal items more effectively. We tested this issue in Experiment 3B.

\section{Experiment 3B}

\section{Method}

Subjects. Twenty undergraduate students attending Simon Fraser University participated in Experiment 3B, for course credit.

Materials. Training stimuli were identical to those of Experiment $3 \mathrm{~A}$. Thus each training item followed the pattern odd-evenodd-even.

We created two sets of test stimuli, one of which was identical to the "familiar bigrams" set used in Experiment $3 \mathrm{~A}$. This set necessarily followed the odd-even rule and so qualified as the "legal" set.

A second set of eight "illegal" stimuli was created by the same bigram-combination rules as the first set; like the first set, these items consisted of familiar bigrams, but presented a novel combination of those bigrams. Each item of this set was then modified by the replacement of one digit by another. In each case, an even digit was replaced by an odd digit, or vice versa. In consequence, the item now violated the odd-even rule at one of its four locations (e.g., 1258 modified to 1268 , producing a violation of the rule in the third location). Violations occurred with equal frequency at each location across the set.

Procedure. The training and test phases were conducted as in Experiment $3 \mathrm{~A}$. Following the test, subjects were interviewed about the basis of their recognition decisions.

\section{Results and Discussion}

The group of subjects who read numbers as lists of individual digits demonstrated sensitivity to the implicit rule (the invariant odd-even pattern) in the recognition test: They claimed to recognize $51 \%$ of legal stimuli and rejected $65 \%$ of illegal stimuli. Their overall discrimination of legal from illegal stimuli was thus $58 \%$, reliably greater than chance $[t(9)=3.28, p<.009]$. Asked how they had performed their recognition judgments, the subjects reported using feelings of familiarity and/or a variety of mini-rules, such as "big numbers at the beginning" or "if it had an eight"; none reported noticing the oddeven pattern that defined legality.

The group of subjects who had read numbers as digit pairs claimed to recognize $60 \%$ of legal items and rejected $80 \%$ of illegal items. Like the first group, they ascribed their judgments to familiarity and to a host of mini-rules unrelated to the actual implicit odd-even rule. Overall, they achieved $70 \%$ discrimination, reliably greater than that of the other group $[t(18)=3.31, p<.004]$.

We thus observed, in both groups, the standard "implicit learning" effect, that subjects can become sensitive to a structural invariant without becoming aware of doing so. However, there is evidence that that sensitivity was not achieved through direct, unconscious abstraction of the invariant during the training, but instead through the encoding of bigrams and use of that knowledge to reject test items containing unfamiliar bigrams. First, according to the automatic abstraction hypothesis, implicit learning is supposed to be unselective and stimulus driven (see discussion after Experiment 1 ). If that were the case, we should observe as much sensitivity to the abstract invariant following the single-digit-encoding task as the bigram-encoding task: Both groups were exposed to the full structure of each item and of the entire domain. Contrary to that hypothesis, the degree of sensitivity to the rule clearly depended on the nature of the induction task: Subjects instructed to read numbers as bigrams achieved considerably greater discrimination between legal and illegal items.

Second, we knew from Experiment $3 \mathrm{~A}$ that the bigram induction task encouraged more extensive encoding of bigrams than did the single-digit task, permitting subjects greater sensitivity in rejecting items containing unfamiliar bigrams. The data of Experiment 3B demonstrated an almost identical pattern of performance: greater discrimination following the bigram induction task and success based more heavily on rejecting items containing unfamiliar bigrams than accepting items containing familiar bigrams. We concluded that subjects in Experiment 3B performed at test in the same way as subjects in Experiment 3A: They claimed to recognize items on the basis of the familiarity of their bigram components and succeeded to the extent that the induction phase had required them to compute such information. Their apparent sensitivity to the implicit invariant was indirect and accidental, resulting from the fact that violations of the rule occurred only in unfamiliar bigrams.

This result demonstrates our central thesis, that the induction task of an implicit learning experiment is not a neutral opportunity to observe and unconsciously abstract the general structure of a domain, thereby becoming implicitly sensitive to its abstract properties. Instead, it clearly demonstrates that the development of sensitivity to implicit aspects of structure is a direct consequence of the demands of the induction task. It further demonstrates that the ability to discriminate between test items that do and do not violate the implicit rule need not be supported by direct knowledge of that rule, but can instead be granted by knowledge correlated with that rule. Finally, it demonstrates that the effective knowledge that permits the subject to show sensitivity to an implicit rule is not the result of unselective, stimulus-driven and autonomous abstraction, but is instead computed directly in response to the demands of the induction task.

On this understanding of implicit learning, during the induction phase subjects only compute information that is relevant to their current purpose for encountering stimuli. In the test phase, the experimenter varies test stimuli along a dimension that subjects cannot anticipate and about which they have no direct knowledge. The subjects apply whatever knowledge they have acquired, and hope for the best. If the knowledge they computed in training is correlated with the dimension manipulated by the experimenter, then their performance will be above chance in the test; if not, they will be at chance on that discrimination. Finding above-chance performance in the test 
phase does not mean that subjects must have directly computed information about the dimension implicitly varied by the experimenter at test; it merely means that they acquired some knowledge, under the control of the induction task, that is correlated with that implicit dimension.

\section{Experiment 4 \\ Implicit Learning of a Nonexistent Invariant}

We believe that the long-standing automatic abstraction hypothesis of implicit learning, and indeed the term "implicit learning" itself, rests on a fallacy of interpretation. In the standard implicit learning experiment, subjects are shown a set of stimuli that possess some abstract, setwise property and are asked to perform some induction task that does not require direct computation of the abstract property. In a subsequent test, subjects are shown items that preserve or violate that property and are observed to discriminate test items of the two kinds, although they cannot report any knowledge of the abstract property. That is, the subjects perform as they would perform if they had learned about the abstract property during the training, and learned it implicitly. The fallacy is to conclude that because they would have performed in that way if they had learned in that way, therefore they did learn in that way. More subtly, the fallacy is to advance this explanation as the default hypothesis, to be accepted unless it can be proven within a particular situation that subjects actually learned and relied on a different form of information.

The object of this experiment was to reverse the onus of proof by demonstrating implicit sensitivity to an abstract property that subjects could not have abstracted during the induction phase. Following a training phase, we showed subjects a set of test items, half of which conformed to the odd-even-odd-even rule used in the last experiment and half of which violated that pattern at some location. Sensitivity to that rule without being able to report it would ordinarily be treated as prima facie evidence of implicit abstraction of the invariant during the training phase. However, in this experiment, that invariant never occurred in any item during the training phase; training items followed a variety of patterns, including odd-even-odd-odd and even-even-odd-even, but none followed the odd-even-odd-even rule. Thus if subjects were able to discriminate between "legal" and "illegal" items at test, we could know that it was not due to implicit abstraction of that rule during training. ${ }^{5}$

However, subjects could discriminate between the two types of test items if they had simply encoded bigrams of training items because "legal" test items were constructed from bigrams used in various training items, whereas both bigrams of "illegal" test items were novel. For example, the bigrams of the "legal" test item 1258 had both been encountered in training, in the items 1257 and 6258, whereas neither bigram of an "illegal" test item such as 1437 had been presented in any training item. Subjects could thus claim to recognize "legal" items by recogniz- ing familiar bigrams and reject "illegal" items by detecting the novelty of their bigrams.

\section{Method}

Subjects. Twelve undergraduate students attending Simon Fraser University participated in Experiment 4, for course credit.

Materials. Sixteen training stimuli were constructed by combining one bigram from the set $\{12,16,34,38,52,58,74,76\}$, in which the first digit was odd and the second even, with one bigram from the set $\{15,24,31,48,57,62,73,86\}$, in which both bigrams were odd or even. Each bigram occurred twice in the training set, once as the first half and once as the second half of a training stimulus. No stimulus contained a repeated digit. Various stimuli presented various combinations of even and odd digits; in each stimulus, there were either three odd digits and one even or alternatively three even and one odd digit. Examples include 1257, 5824, 7312, and 4816. Each item violated the odd-even-odd-even rule at one of its four locations. Across the set, violations occurred at each location in $25 \%$ of items.

Two sets of test stimuli were created. The "legal" set was constructed by combining bigrams from the set $\{12,16,34,38,52,58$, $74,76\}$. Thus each item conformed to the pattern odd-even-oddeven. Each bigram occurred twice in the set, once as the first half and once as the second half of a stimulus (e.g., 1258 and 7612), resulting in eight items. No stimulus contained a repeated digit. Each bigram used to create these items had been presented in two training items (e.g., the 12 in 1258 was presented at training in both 1257 and 7312 )

The "illegal" set was created by combining one bigram from the set $\{13,26,37,42,51,68,75,84\}$ with one bigram from the set $\{14$, $18,32,36,54,56,72,78\}$, none of which occurred in any training item. These bigrams were taken together to form items under the restriction that the resulting item contained three even and one odd or one even and three odd digits. Each bigram occurred once in the set, producing eight items. No stimulus contained a repeated digit.

Both legal and illegal items consisted only of digits that had been presented in training items, and those digits occurred in the same locations in test items in which they had been seen in training items. The test sets could therefore be discriminated only if subjects had learned bigrams of training items.

Procedure. The induction task consisted of reading numbers as pairs of digits; it was identical to the task given to half the subjects in Experiment 3. The test demand, to judge which items they had seen in the training phase, was also identical.

\section{Results and Discussion}

Subjects claimed to recognize $52 \%$ of legal items and rejected $77 \%$ of illegal items. Their overall ability to discriminate items following the odd-even-odd-even rule was thus $64.5 \%$, reliably greater than chance $[t(11)=$ $5.977, p<.001]$. As in Experiment 3B, subjects claimed to have judged the items on the basis of familiarity, coupled with a variety of nondiscriminating mini-rules, such as "if it started with a three."

Thus the subjects were able to discriminate between items that conformed to or violated an abstract structural invariant that did not exist in training items. They obviously did not learn about that invariant directly, either explicitly or implicitly, by abstraction or any other means. Instead, under the demand of the induction task, they learned about the component bigrams of training items, information that we had arranged to be correlated with the implicit odd even rule varied at test. Nevertheless, 
this is an example of "implicit learning": Subjects became sensitive to an abstract property without ever knowing it existed, on the basis of learning about a structurally related set of stimuli under a specific induction task. Moreover, we argue that this is not a special case that artificially meets the definition of "implicit learning." Instead, we argue, it is the paradigm case: Implicit sensitivity to abstract structure occurs when subjects have earlier computed correlated information in order to satisfy the induction task, regardless of whether the abstract property was represented in the training stimuli. The indirect relationship between what the subjects learn in training and the implicit target at test is fundamental to the phenomenon of "implicit learning"; the abstract structure of the training set is not.

We concluded that the encoding of specific knowledge about particular training items, under task control, is a necessary and sufficient explanation of implicit learning. ${ }^{6}$ Together, the experiments in this section demonstrate the bounds of implicit learning. Subjects do not become sensitive to an abstract invariant implicitly present in training stimuli if the induction task leads them to process information unrelated to the invariant (Experiment 2B). They become explicitly aware of the invariant if the task leads them to process information about each stimulus that is directly related to the invariant (Experiment $2 \mathrm{~A}$ ). They become implicitly sensitive to an invariant that is indirectly related to information they are required to process by the specific demands of a task (Experiment 3); but the invariant does not have to be present in the stimuli for them to become sensitive to it at test, as long as the training task causes them to encode aspects of training items that are correlated with the invariant at test (Experiment 4).

We have no doubt that some readers will want to argue that the knowledge that subjects used in Experiments 3 and 4 (the bigrams) is actually abstract knowledge because the bigrams are not identical to the instances from which they are drawn. Further, it might be argued, each bigram occurred in more than one training item, and subjects learned critical bigrams by abstracting these common elements across items. That is, a bigram like 16 consists of a co-occurrence of two features, a 1 and a 6; knowledge of this co-occurrence was strengthened by repetition across instances. Thus the subjects' effective knowledge was actually the result of an abstraction process that is independent of the processing of particular items.

To answer the first, of course the bigrams are abstracted out of items and are therefore abstract knowledge. We do not deny the activity of abstraction, either out of particular instances or across instances (see Whittlesea \& Dorken, 1993, for an example of the latter). However, the abstraction of bigrams out of four-digit numbers was performed deliberately, to satisfy the demand of the task. Our argument is that there is no evidence of abstraction (or any other learning operation) that is performed automatically and unconsciously, independently of the demands of the task. That is, there is no evidence that "implicit learning" is a separate mode of learning, qualitatively different from explicit learning, or that is stimulus driven, unselective, or autonomous. The abstraction of bigrams out of presented items was performed as a direct response to task demands, not as an automatic response to the abstract structure of the domain.

The second idea, that the learning occurred through abstraction of bigrams across items, is just the first idea made more subtle. Our encoding task forced the subjects to treat bigrams, not four-digit numbers, as the unit of processing. They would consequently learn more about a particular bigram on each occasion of encountering it, in the context of different items, just as they would learn more about the face of a person seen in two different photographs. However, to describe that as automatic, unselective, stimulus-driven abstraction across items is clearly wrong: The subjects abstracted each bigram out of each context in which it occurred in response to the demand of the task, not driven by the shared structure of stimuli.

\section{PART III \\ Acquiring the Potential to Learn Later}

Thus far we have been primarily concerned with the induction task and what kinds of implicit and explicit processes occur during that task. However, the test is also an extended processing experience. The automatic abstraction hypothesis treats that task simply as an index of prior learning; having implicitly acquired general, abstract information about the domain during the induction task, the subject simply applies it in test. In contrast, in our account, by processing training stimuli as required by the induction task, people acquire the potential to interact with further stimuli in various ways. They can apply their previous knowledge to discriminate test stimuli, as shown in earlier experiments; but they can also learn during that test. In fact, in the act of applying what they learned about particular stimuli during the training task, subjects may actually be able to learn directly about abstract properties of training set that they did not compute, explicitly or implicitly, during the training.

\section{Experiment 5 Postcomputed Knowledge}

In this experiment, we built into the training stimuli a highly abstract invariant, namely that none of the stimuli presented a regular pattern (e.g., 6945, 1382, and 7436). Unlike the frequency of occurrence of digits in particular locations, or the co-occurrence of digits within items, this irregularity of pattern is a qualitative property that cannot be computed by counting the occurrence of various components across stimuli. In consequence, it is not a property that could be abstracted by a putative automatic frequency-tabulating mechanism. Moreover, the absence of pattern is a negative attribute: Like the absence of beauty or symmetry in a class photograph, it is easy to detect if you are asked to think about the stimulus in terms of that dimension, but is unlikely to be noticed otherwise. 
We therefore suspected that this invariant attribute would not be directly encoded, either implicitly or explicitly, during the training phase.

Moreover, unlike Experiment 4, the test items were not selected to correspond to specific units that subjects had encoded while processing training items, so subjects could not discriminate between test items by recognizing familiar components, as they did in that experiment. Instead, the major difference between sets of test items was that half were irregular and half bore regular patterns. The question was whether subjects could become sensitive to that invariant during the test.

\section{Method}

Subjects. Eighteen undergraduates attending Simon Fraser University participated in this study for course credit.

Materials. We used 30 training items, selected subject to the conditions that none of the items contained any repetition and that none possessed a regular pattern (such as 1234 or 1829). Twelve pairs of further items were generated for test. Each test pair contained a near and a far item. Near test items were generated by changing a single digit of a training item (e.g., the training item 6945 produced the test item 6935). To generate far items, we treated the training items as consisting of a left and a right pair of digits (e.g., 6945 contains the digit pairs 69 and 45). We listed all digit pairs present in the training items and then created the far items from digit pairs not appearing in this list. All far items were then checked to make sure that they were not rearrangements of any single training item (e.g., 9654 would not be allowed because this is a rearrangement of the training item 6945).

The critical manipulation concerned the near items. Six of these items were created to have a pattern that was recognizably regular $(8642,5432,9753,1234,5678$, and 9876), whereas the others did not produce regular patterns $(6935,1546,3721,6725,3514$, and 7681). All far items bore nonregular patterns.

Procedure. Subjects were trained using one of the induction procedures used in Experiment 2: They were asked to compare the sums of the left and the right digit pairs of each training stimulus. They were then given a surprise test involving 12 pairs of four-digit numbers. They were to indicate which of each pair they had seen in the training phase (although all test items were novel). Each test pair contained one near item and one far item; the location of these items within a pair was counterbalanced in a pseudorandom sequence.

\section{Results and Discussion}

When near items did not bear a regular pattern, subjects did not discriminate between near and far items in the recognition task, deciding that the near item was old on $51 \%$ of trials $(S D=21 \%)$, not reliably different from chance $[t(17)<1]$. There was thus no indication that subjects had learned anything about the training stimuli, implicitly or explicitly, that was relevant for making a recognition decision between near and far items.

However, when near items bore regular patterns, subjects did distinguish reliably between near and far items, judging near items to be old on only $34 \%$ of trials $(S D=$ $22 \%$ ), reliably below chance $[t(17)=-2.96, p<.01]$. Clearly, subjects did not perform this judgment using the similarity of surface identities between training and test items because in that case they would have made the opposite judgment, judging near items to be old. Instead, subjects were clearly reacting against these near items because of the regularity of the pattern they carried: Near items were judged old at chance levels when their pattern was irregular, but were judged old reliably less often when their patterns were regular $[t(17)=-2.4, p<.05]$.

Thus subjects judged regular test items to be new. One could argue that, during the induction phase, subjects somehow directly processed the information that all items bore irregular patterns. In principle, it is not possible to disprove this possibility. However, if one wants to imagine that subjects directly worked out, during the training phase, that all stimuli were irregular, then one also has to imagine that they also learned all manner of other properties, which were also invariant during that phase. For example, none of the training stimuli contained letters, were longer or shorter than four digits, contained a repetition, corresponded to important years (e.g., 1945, 1066), summed to 5,000, and so on. The list of absent invariant properties is essentially infinite. Our procedure did not hint that irregularity might be important later, any more than lack of repetition or any of the other potential properties. We therefore suggest that the idea of direct, implicit learning of this property during the training phase is highly implausible.

Instead, we concluded that during training, subjects had implicitly acquired the potential to know that the training items were all irregular, but did not directly learn that knowledge until they encountered regular items in the test; that is, that knowledge was created on the fly (see Kahneman \& Miller, 1986). In encountering the test items, subjects became aware that some were regular. Because they could not recall noticing such a property previously, they concluded that these items must be new. In forming that conclusion, the subjects became sensitive to one of the general, abstract properties of the deep structure of the original domain, that all items carried an irregular pattern. In a sense, that knowledge was acquired during the training phase, through not encountering any regular items--but it existed only in the form of a potential until the regular items were presented in test. At that point, subjects acquired an effective basis of decision that allowed them to reject any item bearing a regular pattern. Equally, they could have become sensitive to any of a variety of other dimensions, such as repetition or stimulus length, had those dimensions been varied in test. Each of these dimensions is implicitly represented across the set of items the subject has already encoded, and each represents a potential form of knowledge. Each becomes realized only when the subject attempts to perform a discrimination along that dimension.

Clearly, this is an extreme case of implicit learning, and one not likely to recommend itself to proponents of the automatic abstraction hypothesis. However, it demonstrates an important point. The knowledge that allows subjects to discriminate between legal and illegal items at test may not have been directly encoded during the original exposure of the items in the training phase; instead, it may have been computed out of that knowledge at the time of test, when items differing on a specific di- 
mension are offered to the subject (see Brooks \& Vokey, 1991; Whittlesea \& Wright, 1997). We argue that subjects learn about the training stimuli in terms of their purpose for encountering them, and in doing so, they acquire the potential to perform many unanticipated tasks, making discriminations along dimensions that were not attended during the original learning. The realization of that potential is a function of the test task, not of the original learning. Knowledge acquired in training is not merely applied in test: Instead, it is modified, interpreted, and elaborated under the demands of the test task and the affordances of the test stimuli, in retroactive comparison with the training stimuli. Under these assumptions, the observation that subjects can discriminate novel stimuli that differ on surface similarity to prior instances or on some more abstract property of the domain cannot be taken as direct evidence that the subjects learned exactly that form of knowledge during the training phase. Assuming that the knowledge controlling test performance is the same as that acquired in learning will underestimate the true complexity of learning and use of knowledge. Although subjects may have acquired some quite definite form of knowledge during training, at test that knowledge base is better understood as a set of resources to be drawn on for many different purposes in interacting with the world.

\section{GENERAL DISCUSSION}

\section{Proximal Versus Distal Sources of Control}

Imagine that a certain person's behavior is strongly sensitive to the lunar cycle. When the moon is nearly full, he is socially active, and when the moon is new, he is reclusive. That is, his behavior is discriminative with respect to the phases of the moon. From observing the state of the moon on a particular day, you can predict his behavior above chance. The question arises of how the moon affects his behavior. When interrogated, the person appears to have no knowledge of the relationship between his behavior patterns and the phase of the moon; the relationship is implicit. Instead, he claims that his social activity is controlled by the amount of money he has on hand. Further investigation reveals that he is paid once every 4 weeks and spends his money as soon as he gets it. Obviously, his financial situation is the direct cause of his behavior.

However, the correlation between his performance and the cycles of the moon is not accidental; historically, that 4-week pay cycle was introduced to reflect the lunar month. Thus the lunar cycle truly is a cause of his behavior pattern, although he is unaware of it. How shall we think of these two sources of control? One is proximal, the other distal; one is explicitly known to the subject, and the other is implicit. Clearly, it is not right to say either that he is insensitive to the lunar cycle, or that it has no effect on his behavior. Rather, its effect is indirect, mediated through the pay schedule and the state of his bank account.

To push the analogy further, the synchronization of this person's behavior with the lunar cycle and the pay schedule seems to suggest that the structure of those cycles exercises unilateral control over his behavior. However, his behavior is in fact the product of adopting a particular strategy for dealing with that structure ("spend it if you have it"). Another person working under the same pay schedule might adopt a different strategy ("save it for a rainy day" or "budget each week"). That person will not exhibit variation in performance correlated with the lunar cycle. However, her performance is constrained by a different aspect of the payment structure--the size of the monthly paycheck. That is, people always have to adapt to the conditions under which they work (frequency and magnitude of payment), but have many options in doing so. Some of these conditions will cause their behavior pattern to be correlated with the underlying structure, and others will not, but in every case, the behavior observed is the product of the person adopting a policy to deal with those important aspects of the environment of which he/she is aware.

In the same way, people in implicit learning experiments are subject to both proximal and distal sources of control. The general, deep, abstract structure of a domain constrains the structure of members of that domain; each individual instance conforms to and exemplifies some aspects of the general structure. People never directly encounter the abstract structure because it is an emergent property of the set taken as a whole. Instead, they only experience the properties of the separate instances. Learning those properties is the proximal cause of later performance differences; the general structure of the domain exercises influence only through constraining the properties of its instances. Moreover, the structure of the stimuli, individually or as a whole, does not constrain people to learn about the stimuli in any particular way; they can adopt a policy of memorizing the instances or computing information about subparts of each stimulus-or even deliberately attempting to count high-frequency features or compounds across successive stimuli. Each of these policies results in a different pattern of later performance in interacting with novel members of the domain; none of them reflects direct sensitivity to the underlying structure of the domain or direct control of performance by the individual structures of the stimuli. Instead, in each case, what subjects learn is dictated by an interaction between their intentions and the structure of the particular instances they encounter, and later performance is controlled by this specific learning in interaction with the structures of test stimuli and the subjects' intentions at the time of test.

\section{Implicit Learning and Implicit Memory}

In parallel with the concept of "implicit learning," there exists another concept called "implicit memory." The literature on this topic focuses on the dissociable effects of a prior experience of a stimulus on performance in a remembering test, like recognition, and a nonreflective task, such as tachistoscopic identification or fragment completion. For example, Jacoby and Dallas (1981) 
observed that a levels-of-processing manipulation of training stimuli influenced the likelihood of claiming to recognize old target words, but not the probability of identifying them. They also observed that prior exposure of a target word at any level of processing reliably facilitated identification. According to separate-systems accounts (e.g., Tulving, 1995), such dissociated effects of prior experience occur because recognition depends on episodic memory, whereas identification is controlled by semantic memory. Episodic memory preserves specific processing and contextual details of the event, causing recognition to be sensitive to the levels manipulation. In contrast, semantic memory contains information about the meaning and physical structure of the item, abstracted across thousands of prior exposures of the word; thus it is not sensitive to context or to the levels manipulation. (To account for the facilitation of identification caused by one extra presentation of a common word, he invoked a third memory system, the PRS.) The abstraction process through which the contents of semantic memory develop is unconscious and automatic; it is driven by the structural identity of the stimulus on each occasion it is experienced, despite differences in the context or task in which the stimulus is encountered.

Clearly, this explanation of implicit memory is very similar to the "abstraction" account of implicit learning described earlier. That account suggests that, in the act of memorizing members of a grammar, episodic information is encoded about the presented items, permitting the person to recognize them later. However, incidental to that task, memory also abstracts general properties of the domain as a whole, knowledge that can later control classification of structurally related but novel members of the category. Thus the major difference between the separate systems accounts of implicit learning and implicit memory is what is to be explained: The facilitated identification of a single previous experience of an item, unaccompanied by recollection of the episodic detail of that experience, or the ability to generalize from a set of structurally related stimuli to novel related items, unaccompanied by awareness of the basis of that performance. In both cases, recollection of the detail of earlier events is dissociated from the effects of those events on nonrecollective performance.

There is an alternative theory of "implicit memory" that does not invoke multiple memory systems - the transfer-appropriate processing theory (Jacoby, 1983; Kolers \& Smythe, 1984; Roediger, Weldon, \& Challis, 1989). The idea of transfer-appropriate processing was originally applied (by Morris, Bransford, \& Franks, 1977) to explain observations that performance on a recognition test depended on the similarity of test conditions to training conditions. More recently, it has been extended to explain dissociations between direct (e.g., recognition) and indirect (e.g., tachistoscopic identification, fragment completion) tests of memory (e.g., Jacoby, 1983; Roediger \& Challis, 1992). The idea is that each of those tests requires certain unique mental operations; either will be successfully performed to the extent that prior experience makes resources available to perform those operations. Roediger and his colleagues (e.g., Roediger \& Challis, 1992) have argued that the usual indirect tests, such as fragment completion, present stimuli in perceptually impoverished ways: A prior experience of an item facilitates performance by providing a directly relevant perceptual representation. In contrast, they have argued, recognition tasks do not present a perceptual challenge: The stimulus is presented in full, for a long duration. Instead, the problem is to discriminate one occurrence of that word (in the context of the experiment) from many other experiences of the same word across the life span. In this case, elaborate, meaningful processing of the training presentation, producing a distinctive representation of that event, assists the person to recognize it later. The transfer-appropriate processing explanation has been able to explain many of the phenomena of "implicit memory" and is at least as successful as the separate-systems account.

The basic assumption of the transfer-appropriate processing account is that success in any test depends on having encoded appropriate resources during the training phase. Those resources are not encoded automatically, as a housekeeping routine of memory. Instead, they are incidental by-products of satisfying the demand of the training task. A subject asked to generate the antonym of hot encodes the meaning of the word cold, but does not experience its visual properties; if instead simply asked to read cold, the subject experiences its perceptual attributes but does not think extensively about its conceptual properties (Jacoby, 1983). Each demand causes the person to selectively process and encode some specific aspects of the stimulus; other aspects, not required to perform the task, remain unprocessed and are not encoded as part of the experience. Neither perceptual nor conceptual information is computed automatically, independently of the demands of the task. Performance in any test task succeeds to the extent that the earlier task performed on that item makes available the resources required by the test. Thus the transfer-appropriate processing account explains performance in both direct and indirect tests of memory through a common set of principles, the assumptions of encoding variability and match of processing between training and test. It denies that chronic abstraction, or a separate representation of episodic and semantic information, is necessary to explain human performance.

This is the same argument that we make in this article concerning the basis of "implicit learning." The training task requires the person to perform specific mental operations on the training stimuli. The training task, whether memorization or any other task, requires the person to process and encode some stimulus attributes; different tasks require the person to process different attributes, and so differentially prepare the person to perform the test task (Experiment 3 ). Those attributes may be unrelated to the resources required to perform the test (Ex- 
periment 2B), in which case performance fails; alternatively, the resource needed in test may be the same as the information that the subject was trying to produce in the training, in which case the person is likely to become aware of using that information (Experiment 2A). However, in satisfying the training task, the person may process and encode some stimulus aspects that are only indirectly related to either the training or the test task (such as encoding bigrams in a whole-item memorization task; Experiments 3 and 4). In that case, they may be able to use that information to perform the task, but be unaware of the source of their success, just as subjects in an "implicit memory" experiment may succeed in completing a word fragment without remembering the experience of that word in the training phase.

\section{Reclaiming the Null Hypothesis}

We do not claim that abstraction, as an automatic, chronic, unconscious activity, is impossible; in fact, numerous investigators have proposed mechanisms by which it might occur (see the introduction). We only argue that there is as yet no evidence that it actually occurs in the human mind. Instead, we argue, all of the evidence currently available about implicit transfer can be explained through the assumptions of encoding variability and match of component processes between training and test. For example, one of the most challenging observations of "implicit learning" is that subjects can be shown training items presented in one set of surface features (e.g., FRXRMMX) and test items in a completely different set (e.g., SQDQLLD) and still discriminate legal test items from illegal ones (see, e.g., Brooks \& Vokey, 1991; Howard \& Ballas, 1982; Manza \& Reber, 1992; Mathews, Buss, et al., 1989). This seems to be direct evidence that the knowledge used to discriminate test items is about the deep rather than the surface structure of the training set; it further suggests the operation of an abstraction mechanism operating automatically on the deep structure of the set while the subjects attempt to memorize the surface structures of the training items.

However, this evidence was obtained in studies that used a classification test. Necessarily, the subjects had to be informed at test that the training stimuli followed rules and that test stimuli followed or violated those rules. Brooks and Vokey (1991) pointed out that this information permitted subjects who had not abstracted any knowledge about deep structure during training to compute some aspects of that structure during the test. For example, a subject faced with the novel legal item SQDQTD might succeed in recalling all or part of the training item FRXRMMX. If she did so, she could then analyze the repetition pattern of that item, deliberately and consciously, and correctly judge SQDQTD to be legal. Thus, in principle, the observation of success in discriminating changedfeatures items in a classification task need not mean that people automatically abstract deep structure.
The problem of alerting subjects to the existence of rules can be avoided by the use of indirect tests, such as pleasantness judgments, in which subjects do not have to be informed of the relationship between training and test. Gordon and Holyoak (1983) demonstrated that subjects discriminate between legal and illegal test items in pleasantness ratings much as they do in classification. However, in their experiment, test items were presented in the same set of features as training items, which could itself cue subjects to use information about the training set. Whittlesea and Wright (1997) combined the two ideas: We presented legal and illegal test items in a novel set of surface features in a pleasantness-rating task. In this test, neither the task nor the surface features corresponded to the experience of training items; however, the legal test items preserved the deep structure of the training set. If deep structure is automatically abstracted from training items and applied automatically to further items bearing the same structure, performance should be above chance. However, performance was at chance. The same subjects were then given a classification test that required explaining the relationship between training and test phases. In this test, subjects were regularly observed to perform above chance. We concluded that when subjects are not made aware of the structural relationship between training and test stimuli, either through instruction or use of the same feature set, there is no evidence of automatic sensitivity to the deep structure of the training domain. Instead, we concluded, the subjects' sensitivity to deep structure in classification resulted from deliberately abstracting pattern information from remembered instances at the time of test, reacting to the instructions of that test, rather than being automatically and unconsciously abstracted during the training (see Experiment 5).

We argue that the abstraction hypothesis, although a possible explanation of "implicit learning," has the status of a speculation, rather than of well-founded theory. We are not aware of any critical evidence, in any domain or area, that the human mind possesses a mechanism that automatically computes abstract properties across experiences. In contrast, the principles that we have used to explain implicit sensitivity to structure (encoding variability and the overlap of processing between training and test) are widely established as the fundamental principles controlling performance in direct tests of memory for single prior experiences and are at least in the running as the principles to explain indirect tests of single experiences. We invoke no further processing assumptions to explain performance in direct and indirect tests of memory for multiple related stimuli; we have simply demonstrated how those same assumptions can explain the phenomena of implicit as well as explicit transfer of knowledge. We therefore argue that there is no need to proliferate memory entities or modes of learning. Until some new form of evidence, currently not available, makes unconscious abstraction a necessary construct, we should treat the idea that memory depends on the same principles in explicit and implicit tests as the null hypothesis. 


\section{What's in a Phrase? The Ambiguity of "Implicit Learning"}

The term "implicit learning" has suffered from an ambiguity. It actually refers to an effect, an observation that people can become sensitive to implicit properties without becoming aware of those properties. However, naming the effect in this way presupposes a specific theoretical interpretation of the effect. The words seem to mean that the subject has actually learned the implicit property itself. This initial characterization of the phenomenon led to all of the additional assumptions of abstraction theory: To become sensitive to an abstract, implicit property without awareness must mean there are two modes of learning, one explicit and one implicit; that the latter must be automatic and unselective because it is not modulated by intention to learn about that property; and that that learning must occur through an act of abstraction because the subject is later sensitive to an abstract property. However, the phenomenon itself does not entail any of these assumptions.

We claim that people directly acquire information only about those stimulus aspects they are required to process, under the demands of the task, but in doing so acquire the potential to respond along unanticipated dimensions. They are learning without awareness, but without awareness of the consequences of their current behavior, not of what they are currently learning, or their current intentions, or the demands under which they learn. They have learned something that makes them sensitive to implicit properties, but to call that "implicit learning" is parallel to referring to the act of winning a lottery as "implicit spending." The one action enables the other, but the relationship is indirect. We would prefer that the phenomenon had been named in more neutral terms, such as "acquisition of sensitivity to implicit properties." Although a less handy phrase, it is a clearer description of the phenomenon itself, independently of any theoretical account. The chief obstacle in understanding "implicit learning" has been the name itself.

\section{Learning Without Knowing the Consequences: An Integrative Account}

We argue that all learning, whether accompanied by awareness of some domainwide organization or not, occurs when a person encounters a stimulus in some context and for some purpose (see Whittlesea \& Dorken, 1993). The purpose and context, together with the person's past learning history with similar stimuli, dictate the operations the person performs on the stimulus. Memory preserves the experience of processing the stimulus in that way; the representation of that experience can control performance toward similar stimuli on subsequent occasions. Consciousness of the existence or importance of some particular deep or surface property of the stimuli may alter the processing performed by changing the person's purpose for encountering the stimulus, but it is not the sole or dominant agent responsible for variations in processing.
Subjects in implicit learning experiments are always actively involved with the stimuli, for one purpose or another. They are variously asked to observe (e.g., Gordon \& Holyoak, 1983), memorize (e.g., Reber \& Allen, 1978), or perform computations on the stimuli (e.g., McGeorge \& Burton, 1990). What they are required to do dictates what resources they will bring to bear on the stimuli, and in consequence how they will process those stimuli. Differences in these activities will produce differences in what subjects learn, independently of the structure of the domain. Moreover, the subject is not even a passive performer of the task demanded by the experimenter. Asked to memorize the stimuli, subjects make assumptions about what is important in the environment and how to operate on it and respond to it: They exercise various strategies of memorization, adapting their performance to the peculiarities of the stimuli created by the experimenter (see, e.g., Jacoby \& Bartz, 1972). These decisions determine what aspects of the structure they will attend and how they will organize those aspects into mental structures. The learning performed when unaware of general structure is an active adaptation to circumstances, just as much as is explicit hypothesis testing.

The importance of active adaptation in determining the course of implicit learning, and the variability in learning that it can produce, has been underrated because the procedure of standard implicit learning experiments reduces it to a minimum. The subject has to be allowed to do something with the stimuli, or else no learning is observed. However, the subject's purpose for encountering the stimuli (the orientation task) is held constant, discouraging variability of processing, and the subject's involvement with the stimuli (the elaborateness of processing) is purposely kept low to avoid deliberate hypothesis testing. In fact, these characteristics are unrepresentative of implicit learning in natural situations. Ordinarily, in interacting with examples of a natural domain, such as language, people have a wide variety of purposes and tasks, and those tasks involve them in extensive processing. The fact that subjects do not display much variability in performance in standard implicit learning experiments does not mean that they learn passively. It merely means that their behavior is constrained by the limited opportunity to process stimuli in different ways.

We conclude that when subjects are exposed to a domain containing deep structure, but are unaware that such structure exists, the knowledge they acquire that makes them sensitive to the deep structure is a by-product of the processes they apply to stimuli in order to perform the task they are aware of, namely the induction task. This conclusion contradicts the idea that implicit learning is stable, or unselective, or driven only by the structure of the stimuli. Instead, it suggests that implicit learning, like explicit learning, is the product of applying specific processes to stimuli, processes that are selected by the task in interaction with memory for experiences of performing similar tasks or working with similar stimuli. These processes can be highly variable, and that variability will 
be controlled as much by the purpose for encountering the stimuli as by the structure of the stimuli and will lead to quite different aspects of stimulus structure being encoded in memory.

Thus the only major difference between implicit and explicit learning may be that consciously knowing that a domain possesses some important structural property can cause one to try to learn specifically about that property, whereas the processing performed when unaware that such a property exists may focus selectively on less relevant properties. Further, subjects knowing that there is a deep structure can evaluate whether and how much they are learning by their success in predicting members of the domain, whereas subjects processing the stimuli for a different purpose may not feel that they are learning at all because they are unaware that their current activity is relevant to any future behavior. However, the processing can be just as flexible, selective, and variable when one is or is not aware of such a critical property, and in fact proceeds in the same fundamental way. People process stimuli in whatever way serves to meet the demands of the current task, given the memorial resources available and their understanding of the current task. That people reap unanticipated benefits in processing structurally related stimuli on subsequent occasions is not a reason to believe that they originally learned directly, but unconsciously, about that particular structure.

\section{REFERENCES}

Berry, D. C., \& Broadbent, D. E. (1988). Interactive tasks and the implicit-explicit distinction. British Journal of Psychology, 79 , 251-272.

BERRY, D. C., \& DiENES, Z. (1993). Implicit learning: Theoretical and empirical issues. Hillsdale, NJ: Erlbaum.

BROOKs, L. R. (1978). Non-analytic concept formation and memory for instances. In E. H. Rosch \& B. B. Lloyd (Eds.), Cognition and categorization (pp. 169-211). Hillsdale, NJ: Erlbaum.

BROOKs, L. R. (1987). Decentralized control of categorization: The role of prior processing episodes. In U. Neisser (Ed.), Concepts and conceptual development: Ecological and intellectual factors in categorization (pp. 141-174). Cambridge: Cambridge University Press.

BROOKS, L. R., \& VOKEY, J. R. (1991). Abstract analogies and abstracted grammars: Comments on Reber (1989) and Mathews et al. (1989). Journal of Experimental Psychology: General, 120, 316-323.

Cleeremans, A. (1993). Mechanisms of implicit learning: Connectionist models of sequence processing. Cambridge, MA: MIT Press.

COCK, J. J., BERRY, D. C., \& GAFFAN, E. A. (1994). New strings for old: The role of similarity processing in an incidental learning task. Quarterly Journal of Experimental Psychology, 47A, 1015-1034.

DienEs, Z. (1992). Connnectionist and memory array models of artificial grammar learning. Cognitive Science, 16, 41-79.

Dulany, D. E., Carlson, R. A., \& Dewey, G. I. (1984). A case of syntactical learning and judgment: How conscious and how abstract? Journal of Experimental Psychology: General, 113, 541-555.

GORDON, P. C., \& HOLYOAK, K. J. (1983). Implicit learning and generalization of the "mere exposure" effect. Journal of Personality \& Social Psychology, 45, 492-500.

Hayes, N., \& Broadbent, D. E. (1988). Two modes of learning for interactive tasks. Cognition, 28, 249-276.

Howard, J. H., \& Ballas, J. A. (1982). Acquisition of acoustic pattern categories by exemplar observation. Organizational Behavior \& $\mathrm{Hu}$ man Performance, 30, 157-182.

JACOBY, L. L. (1983). Remembering the data: Analyzing interactive processes in reading. Journal of Verbal Learning \& Verbal Behavior, $22,485-508$
JACOBY, L. L., \& BARTZ, W. H. (1972). Encoding processes and the neg. ative recency effect. Journal of Verbal Learning \& Verbal Behavior, 11, 561-565.

JACOBY, L. L., \& DALLAS, M. (1981). On the relationship between autobiographical memory and perceptual learning. Journal of Experimental Psychology: General, 110, 306-340.

Kahneman, D., \& Miller, D. T. (1986). Norm theory: Comparing reality to its alternatives. Psychological Review, 93, 136-153.

KNOWLTON, B. J., \& SQUIRE, L. R. (1994). The information acquired during artificial grammar learning. Journal of Experimental Psychology: Learning, Memory, \& Cognition, 20, 79-91.

Kolers, P. A., \& SMYTHE, W. E. (1984). Symbol manipulation: Alternatives to the computational view. Journal of Verbal Learning \& Verbal Behavior, 21, 289-314.

LEWICKI, P. (1986). Nonconscious social information processing. New York: Academic Press.

LEWICKI, P., \& HILL, T. (1989). On the status of nonconscious processes in human cognition: Comment on Reber. Journal of Experimental Psychology: General, 118, 239-241.

MANZA, L., \& REBER, A. S. (1992). Inter- and intra-modal transfer of an implicitly acquired rule system. Unpublished manuscript, Brooklyn City College.

Mathews, R. C., Buss, R. R., Stanley, W. B., BlanChard-Fields, F., Cho, J. R., \& Druhan, B. (1989). Role of implicit and explicit processes in learning from examples: A synergistic effect. Journal of Experimental Psychology: Learning, Memory, \& Cognition, 15, 1083-1100.

Mathews, R. C., Druhan, B. B., \& Roussel, L. G. (1989, November). Forgetting is learning: Evaluation of three induction algorithms for learning artificial grammar. Paper presented at the annual meeting of the Psychonomic Society, Boston.

Mathews, R. C., \& Roussel, L. G. (1993). Automatic abstraction of stimulus structure from episodes: Comment on Whittlesea and Dorken. Journal of Experimental Psychology: General, 122, 397-400.

MCGEORGE, P., \& BURTON, A. M. (1990). Semantic processing in an incidental learning task. Quarterly Journal of Experimental Psychology, 42, 597-610.

MorRIS, C. D., BRANSFord, J. D., \& FranKs, J. J. (1977). Levels of processing versus transfer-appropriate processing. Journal of Verbal Learning \& Verbal Behavior, 16, 519-533.

NisSEN, M. J., \& BULLEMER, P. (1987). Attentional requirements of learning: Evidence from performance measures. Cognitive Psychology, 19, 1-32.

Perruchet, P., \& Pacreau, C. (1990). Synthetic grammar learning: Implicit rule abstraction or explicit fragmentary knowledge? Journal of Experimental Psychology: General, 119, 264-275.

Perruchet, P., \& Pacteau, C. (1991). The implicit acquisition of abstract knowledge about artificial grammar: Some methodological and conceptual issues. Journal of Experimental Psychology: General, 120, 112-116.

REBER, A. S. (1969). Transfer of syntactic structure in synthetic languages. Journal of Experimental Psychology, 81, 115-119.

REBER, A. S. (1976). Implicit learning of synthetic languages: The role of instructional set. Journal of Experimental Psychology: Human Learning \& Memory, 2, 88-94.

REBER, A. S. (1989). Implicit learning and tacit knowledge. Journal of Experimental Psychology: General, 118, 219-235.

REBER, A. S. (1993). Implicit learning and tacit knowledge: An essay on the cognitive unconscious. New York: Oxford University Press.

REBER, A. S., \& ALLEN, R. (1978). Analogic and abstraction strategies in synthetic grammar learning: A functionalist interpretation. Cognition, 6, 193-221.

REBER, A. S., \& LEWIS, S. (1997). Toward a theory of implicit learning: The analysis of the form and structure of a body of tacit knowledge Cognition, 5, 333-361

RoEdiger, H. L., III, \& Challis, B. H. (1992). Effects of exact repetition and conceptual repetition on free recall and primed word fragment completion. Journal of Experimental Psychology: Learning. Memory, \& Cognition, 18, 3-14.

Roediger, H. L., III, Weldon, M. S., \& Challis, B. H. (1989). Explaining dissociations between implicit and explicit measures of retention: A processing account. In H. L. Roediger III \& F. I. M. Craik 
(Eds.), Varieties of memory and consciousness: Essays in honour of Endel Tulving (pp. 3-41). Hillsdale, NJ: Erlbaum.

Servan-Schreiber, E., \& Anderson, J. R. (1990). Learning artificial grammars with competitive chunking. Journal of Experimental Psychology: Learning, Memory, \& Cognition, 16, 592-608.

Tulving, E. (1995). Organization of memory: Quo vadis? In M. Gazzaniga (Ed.), The cognitive neurosciences (pp. 839-847). Cambridge, MA: MIT Press.

VoKEY, J. R., \& BRooks, L. R. (1992). Salience of item knowledge in learning artificial grammars. Journal of Experimental Psychology: Learning, Memory, \& Cognition, 18, 328-344.

WhitTleseA, B. W. A., \& Dorken, M. D. (1993). Incidentally, things in general are particularly determined: An episodic-processing account of implicit learning. Journal of Experimental Psychology. General, 122, 227-248.

WhitTLeseA, B. W. A., \& WRIGHT, R. L. (1997). Implicit (and explicit) learning: Acting adaptively without knowing the consequences. Journal of Experimental Psychology: Learning, Memory, \& Cognition, 23, 181-200.

Wright, R. L., \& BurTon, A. M. (1995). Implicit learning of an invariant: Just say no. Quarterly Journal of Experimental Psychology, 48, 783-796.

\section{NOTES}

1. The use of a recognition rather than classification as a measure of sensitivity to abstract structure may surprise some readers. However, recognition is one of several indirect measures commonly used to assess "implicit learning"; others include pleasantness judgments (Gordon \& Holyoak, 1983) and anagram solving (Reber \& Lewis, 1977). Unlike classification, such indirect tests allow one to present legal and illegal items to subjects without making them aware that the domain has a deep structure. As long as all test items are novel, recognition and classification tests produce substantially similar performance (Whittlesea \& Dorken, 1993).

2. However, there is evidence that this task caused subjects to encode information of indirect relevance to the invariant 3 and that this was the actual basis of the later sensitivity. First, Cock et al. (1994) performed a series of experiments using the McGeorge and Burton (1990) "invariant 3" paradigm, using an arithmetical orientation task in which subjects added together the left pair of digits, and separately the right pair of digits, of each training stimulus. Their test stimuli were selected to vary on two dimensions, the possession of a 3 and the similarity of the whole stimulus to some training stimulus. They concluded that subjects could discriminate "legal" from "illegal" items in test, but that the basis of that ability was not abstraction of the "invariant 3." Instead they concluded that the effective basis of decision was the similarity of test instances to particular training instances, and that learning in this task mainly consists of the encoding of training instances. Second, knowledge concerning the sums of the "left" and "right" numbers may be useful at test because the presence of the invariant 3 in the positive test items affects the sums of the digit pairs (Wright \& Burton, 1995). Its presence will mean that the sums of the pairs contained in the positive items will, on average, be lower than the sums of the negative items (if it was instead an "invariant 9" task, then the sums of the positive items would be generally higher than the sums of the negative items). In fact, the sums of the positive items will be more similar to the sums of the positive items seen during training, which also contain a 3 . A subject using analogic processing on the basis of sums would be more likely to select a positive than a negative item.

3. We do not mean that people are necessarily aware of what they learn. For example, in reading the word TIGER and judging it to be similar to LION, I am aware of the meanings of the words, but not aware that I just acquired a representation of the orthographic (structural) properties of TIGER that will later assist me in completing TIG__ in a later test.
That aspect of the learning is implicit, but it is not an unconscious abstraction across stimuli. Second, that set of properties is learned because I actually had to process them in order to identify the word; if instead I heard the word, its visual properties would not be processed or encoded (see Jacoby, 1983). Third, people may encode salient properties that are absolutely irrelevant to their task: If TIGER accidentally follows PAPER in a task of judging word frequency, subjects may notice and encode the coincidental PAPER TIGER. Again, however, this is not chronic, automatic abstraction of general structure. Finally, because of the breadth of properties that one could in principle abstract from a domain like the present one, no experiment could prove that people do not abstract some domainwide properties. For example, in our study, they might have been implicitly computing the difference of the first pair but not the other pairs, or the frequency of numerals in location. However, we argue that there is as yet no demonstration of people directly computing an abstract, general property of a stimulus domain that is unrelated to knowledge they were led to process by the overt task. We discuss this further in the General Discussion.

4. Our subjects apparently had a natural tendency to parse and encode large numbers as bigrams. In initial studies, we asked subjects to read four-digit numbers as a series of four digits without performing the high-low decision. In that case, there was no difference between that group and the group reading numbers as bigrams on the recognition test. However, we also observed that they broke the numbers into two bigram pairs in spite of naming the digits separately; they spoke rhythmically, saying, for example, "1-4, [pause], 5-8." We concluded that those subjects had effectively treated the numbers as consisting of two bigrams. It was to prevent this parsing that we added the extra high-low judgment in the single-digit training.

5. Mathews and Roussel (1993) argued that abstraction of structure is automatic and unconscious, but acts only on the structure of the stimuli as the subject processed them for the required purpose, rather than reacting to the objective structure of the domain. That interpretation could explain the results of our Experiment 3. However, it could not explain the results of this experiment: Every item, and $50 \%$ of the bigrams (one in each item) violated the rule. In consequence, if subjects had encoded the stimuli as processed under the demands of the task, they would have encoded as many bigrams violating the rule as bigrams preserving the rule. If they then proceeded to abstract the pattern of the bigrams, they would have computed the information that the bigrams were odd-odd or even-even as often as they were odd-even, and that each item possessed one bigram of each type. That information would not render subjects sensitive to the even-odd-even-odd rule imposed at test. If anything, it should have made them prefer the "illegal" items because those items, like the training items, consisted of one odd-even bigram and one odd-odd or even-even bigram. That is, the abstract structure of the "illegal" items actually matched the abstract structure of training items more closely than that of the "legal" items did. Our subjects' preference for "legal" items at test indicated that they actually preserved and used specific legal bigrams presented in training, not abstractions based on those bigrams

6 . We should point out that implicit learning is not necessarily mediated by the encoding of bigrams; we concentrated on them in these studies for convenience. In other studies, we have demonstrated that sensitivity to an implicit property can be mediated by coding entire instances or by abstracting repeated elements and by processing stimuli alternatively for their orthographic or phonological properties (Whittlesea \& Dorken, 1993). However, we believe that the principle of learning, under task control, about item properties that are correlated with an unanticipated dimension is the general explanation of the implicit development of sensitivity to abstract properties of the domain.

(Manuscript received November 7, 1996; revision accepted for publication January 30, 1997.) 